\title{
New Ways to Look at Blood Pressure
}

\author{
Shah Murad ${ }^{1 *}$, Adnan Shafique ${ }^{2}$, Amarlal Ghurbakhshani ${ }^{3}$, Seema Shah Murad $^{4}$, Abdul Ghaffar ${ }^{5}$ and Jamila Shah $^{6}$ \\ ${ }^{1}$ Department of Pharmacology, Islamabad, Pakistan \\ ${ }^{2}$ Clinical Pharmacist at MCC Pharmacy, ISD, Pakistan
}

${ }^{3}$ Department of Physiology at CMC Larkana Sindh, Pakistan

${ }^{4}$ Department of Gynecologist at NMC, Karachi, Pakistan

${ }^{5}$ CWO at IMDC and DANTH, Islamabad, Pakistan

${ }^{6}$ Department of Psychologist at BU Karachi Pakistan

*Corresponding author: Shah Murad, Department of Pharmacology, IMDC, Islamabad, Pakistan

\begin{abstract}
There are ethnic-based medicinal herbs which can be used to treat or prevent CAD with narrow to moderate range of statistical significance. We compared Rosuvastatin $10 \mathrm{mg}$ with Indian dates (Jujubes) as hypolipidemic agents. This research was conducted at Ghurki Trust Teaching Hospital, jalo park, Lahore, Pakistan. Duration of study was two months. Twenty to seventy years old 60 hyperlipidemic patients of both genders were included in research work with written consent. We divided these patients in two equal groups. Group-I was advised to take Tablet Rosuvastatin $10 \mathrm{mg}$, twice daily for two months. Group-II was advised to take 500 grams Indian dates for two months. Their baseline parameters like LDL-cholesterol, HDL-cholesterol, systolic/diastolic blood pressure was determined in the hospital laboratory. Separate file was maintained for their name, age, sex, occupation, and address. After two months therapy we compiled data related to tested parameters. Paired t-test was applied to compare changes in all parameters. Their mean values with \pm SD before and after treatment were compared and analyzed statistically. It was observed that Rosuvastatin significantly decreased systolic/diastolic blood pressure, LDL-cholesterol, and increased HDL-cholesterol in 27 hyperlipidemic/hypertensive patients. Indian dates used in 30 hyperlipidemic patients significantly decreased systolic blood pressure, and LDL-cholesterol, but insignificant changes were seen in diastolic blood pressure, and HDL-cholesterol. We concluded from the research work that Rosuvastatin is potent hypolipidemic and hypotensive medicine as compared to indian dates.
\end{abstract}

\section{Introduction}

With a low calorie count and a higher protein and fiber level, indian date helps to satisfy nutritional needs and fill up, which prevent from snacking in between meals. This will help maintain diet or prevent any additional weight gain [1-10]. Indian dates i.e. Jujube is one of the good sources of antioxidant content, like vitamin C, vitamin A, and numerous organic compounds. Antioxidants help to neutralize free radicals, the dangerous byproducts of cellular respiration, which are liable for several chronic diseases and illness within the body. Vitamin C also encourages the production of white blood cells, the first line of defense of human body immune system $[11,12]$. The use of statin agents in patients with acute coronary syndromes (ACSs) remains an area of intense clinical interest [13]. Statin therapy has an established secondary preventive benefit in patients with coronary artery disease, and its extension to acute coronary syndrome seems logical [14]. A number of observational studies have shown an association between initiation of statin therapy early in acute coronary syndrome and improved clinical outcome. Four randomized controlled trials have examined the use of statin therapy for acute coronary syndrome: The Myocardial Ischemia Reduction with Aggressive Cholesterol Lowering study, the Pravastatin Turkish Trial, the Rosuvastatin on Risk Diminishing After Acute Myocardial Infarction study, and the Lipid-Coronary Artery Disease study. Three of these trials showed a benefit with early initiation of statin therapy, whereas one trial demonstrated neither benefit nor harm [15]. To reversing the inhibitory effect of oxidized LDL on nitric oxide synthase-3, Rusuvastatin also have direct antioxidant effects on LDL in vitro and ex vivo. Metabolites of Rusuvastatin, but not the parent compound, inhibit oxidation of both LDL and very-low-density lipoprotein as well as highdensity lipoprotein [16]. Metabolites, representing 70\% of active Rusuvastatin in plasma, demonstrate free radical-scavenging abilities that may contribute to inhibition of lipoprotein oxidation [17]. Rusuvastatin also indirectly affect normal oxidative 
mechanisms by curbing the ability of macrophages to oxidize lipoproteins [18].

\section{Subjects and Method}

The research was conducted at Ghurki Trust Teaching Hospital, lahore-pakistan from January to June 2019. Sixty primary and secondary hyperlipidemic and hypertensive patients were selected from Ghurki Trust Teaching Hospital, Lahore, Pakistan. The research aim was to compare hypolipidemic and hypertensive effects of Rosuvastatin $10 \mathrm{mg}$ and Ziziphus Jujubes (Indian dates) in these patients. Both male and female patients suffering from primary or secondary hyperlipidemia were selected. The age limit for patients was 20 to 70 years. Patients suffering from any major organ disease like liver, lungs, kidney, thyroid, heart and eye complications were excluded from the research. Written consent was taken from all participants. Baseline Lipid Profile was determined in Biochemistry lab of the Hospital. Serum cholesterol was estimated by enzymatic method using kit Cat. No: 303113050 by Eli Tech Diagnostic, France. Serum HDL-cholesterol was determined by using kit Cat No: 303210040 by Elli Tech Diagnostic, France. Chylomicrons, low density lipoprotein and very low density lipoprotein are specially precipitated with phosphotungstic acid and magnesium ions can then be removed by centrifugation, while high density lipoproteins remain in the supernatant. Cholesterol included in this phase is measured by an enzymatic method. LDL-cholesterol was calculated according to Friedwald formula [16] i.e. LDL= TC-(TG/5+ HDL-C).
All Patients were divided in two groups, 3o patients in each group. Group-I was on Tablet Rosuvastatin $10 \mathrm{mg}$ twice daily for two months. Group-II was on Jujube 500 grams daily in three divided times to eat. They were advised to take this fruit for two months. Mean values \pm SD were taken for statistical analysis. For parallel comparison, we used paired ' $\mathrm{t}$ ' test to get significance changes in tested parameters at start of treatment and at end of the research work. P-value $>0.05$ was considered as non-significant change, p-value $<0.01$ was considered as significant and $p$-value $<0.001$ was considered as highly significant change in the tested parameter. We used SPSS version 2010 for statistical analysis.

\section{Results}

HMG-CoA reductase inhibitor (Rosuvastatin $10 \mathrm{mg}$ ) when used for two months in 27 hyperlipidemic patients, it reduced systolic blood pressure $30.1 \mathrm{~mm}$ of mercury and diastolic blood pressure $9.7 \mathrm{~mm}$ of mercury, LDL-C $29.2 \mathrm{mg} / \mathrm{dl}$, and increased HDL-C 7.3 $\mathrm{mg} / \mathrm{dl}$. In group-II $(\mathrm{n}=30)$ which was advised to take Indian dates for two months, it reduced systolic blood pressure $10.9 \mathrm{~mm}$ of mercury, diastolic blood pressure $5.1 \mathrm{~mm}$ of mercury, LDL-C 7.9 $\mathrm{mg} / \mathrm{dl}$ and increased HDL-C $3.3 \mathrm{mg} / \mathrm{dl}$. Changes in all parameters are shown in Table $1 \& 2$. SBP means systolic blood pressure, DBP means diastolic blood pressure measured in mm of mercury, LDL-C means low density lipoprotein cholesterol, HDL-C means high density lipoprotein cholesterol measured in $\mathrm{mg} / \mathrm{dl}$. ' $\mathrm{n}$ ' means sample size.

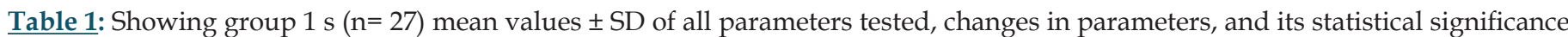
in change.

\begin{tabular}{|c|c|c|c|c|}
\hline Parameter & At Starting of Treatment & After Two Months & Change in Parameter & Statistical Significance (P-Value) \\
\hline SBP & $150.22 \pm 1.11$ & $120.11 \pm 1.91$ & 30.1 & $<0.001$ \\
\hline DBP & $97.91 \pm 1.21$ & $88.21 \pm 1.11$ & 9.7 & $<0.01$ \\
\hline LDL-C & $210.16 \pm 2.11$ & $180.97 \pm 2.22$ & 29.2 & $<0.001$ \\
\hline HDL-C & $37.91 \pm 1.91$ & $45.21 \pm 2.19$ & 7.3 & $<0.01$ \\
\hline
\end{tabular}

Table 2: Showing group $2 \mathrm{~s}(\mathrm{n}=30)$ mean values \pm SD of all parameters tested, changes in parameters, and its statistical significance in change.

\begin{tabular}{|c|c|c|c|c|}
\hline Parameter & At Starting of Treatment & After Two Months & Change in Parameter & Statistical Significance (P-Value) \\
\hline SBP & $141.71 \pm 2.21$ & $130.78 \pm 1.11$ & 10.9 & $<0.01$ \\
\hline DBP & $93.61 \pm 2.00$ & $88.54 \pm 1.10$ & 5.1 & $>0.05$ \\
\hline LDL-C & $198.82 \pm 2.17$ & $190.91 \pm 1.73$ & 7.9 & $<0.01$ \\
\hline HDL-C & $38.61 \pm 2.19$ & $41.91 \pm 2.97$ & 3.3 & $>0.05$ \\
\hline
\end{tabular}

\section{Discussion}

In our results two months therapy by Rosuvastatin $10 \mathrm{mg}$ when used in 27 hyperlipidemic patients, it affected, when statistically analyzed, all tested parameters included systolic/diastolic blood pressure, LDL-cholesterol and HDL-cholesterol. Indian dates proved no significant changes in 30 hyperlipidemic patients in their diastolic blood pressure and HDL-cholesterol, but did affect systolic blood pressure and LDL-cholesterol significantly, with p-values $<0.01$. Bihva C et al. [19] explained same mechanism of action of Rosuvastatin as described in textbooks of medicines, pharmacology and therapeutics that it inhibits HMG-CoA reductase enzyme which is responsible to synthesize cholesterol in human body. They proved same effects of this drug on 56 hyperlipidemic patients. Cella V et al. [20] proved $30.99 \mathrm{mg} / \mathrm{dl}$ reduction in LDLcholesterol when they used Rosuvastatin $10 \mathrm{mg}$ once daily for three months in 109 hyperlipidemic patients. Mekatal Y et al. [21] said in their conclusion that statins are the best among hypolipidemic agents used in patients suffering from primary or secondary hyperlipidemia. Ketylu V et al. [22] emphasized to use Rosuvastatin in those patients who are victimized by metabolic syndrome with increased oxidative stress causing lethality in these patients due to myocardial infarction. Burden of free radical formation, diabetes, obesity, hypertension, hypo or hyperthyroidism, excessive inflammatory reactions in body, and utilization of fatty foods may cause, rather do cause coronary artery syndrome, which is 
difficult to treat, but not impossible. Statins like Rosuvastatin is the best example of drugs used in these patients [23]. Kakati PY et al. [24] have provided other options of treating patients suffering from hyperlipidemia, other than allopathic drug regimens. They recommended herbal medicines or medicinal plants to treat complicated cases of hyperlipidemia. They used Indian dates in 46 hyperlipidemic patients one kg daily for three days and proved LDL-cholesterol reduction $8 \mathrm{mg} / \mathrm{dl}$. No HDL-cholesterol increase was seen by them. Lomateevasel IO et al. [25] proved $20.6 \mathrm{mg} / \mathrm{dl}$ reduction in LDL-cholesterol when 400 grams indian dates were used in 22 hyperlipidemic patients for two months. They also proved reduction in blood pressure significantly in their patients. Blood pressure significant effect is not proved in many studies conducted on indian dates [26].

\section{References}

1. Zito F, Lowe GD, Rumley A, McMahon AD, Humphries SE (2013) Z Jujubes are hypoglycemic.Atherosclerosis 165: 153-158.

2. Ahmed I, Adeghate E, Sharma AK, Pallot DJ, Singh P (2015) Jujubes in indian culture and health related problems. Diabetes Research and Clinical Practice 40: 145-51.

3. Mulder AB, van den Bergh FA, Vermes I (2013) Medicinal ingredients in $\mathrm{Z}$ jujubes. Clin Pharmacol Ther 73: 475.

4. Garcia PJ (2015) Herbal medicine for hundred of diseases. Curr Atheroscler Rep 7: 34-39.

5. Atole SK, Jangde CR, Philip P, Rekhe DS, Aghav DV, et al. (2013) Safety Evaluation Studies of Indian dates. Pharma World 2: 423-425.

6. Chaves FJ, Real JT, Garcia-Garcia AB, Civera M, Armengod ME, et al. (2014) Z Jujubes for hyperlipidemia. J Clin Endocrinol Metab 86: 4926 4932.

7. Mulder AB, van Lijf HJ, Bon MA, van den Bergh FA, Touw DJ, et al. (2016) Z Jujubes (indian dates) are harmless hypoglycemic agents. Clin Pharmacol Ther 70(2): 546-551.

8. Ghirlanda G, Oradei A, Manto A, Lippa S, Uccioli L, Caputo S et al. (2015) Indian dates are getting popularity in phytotherapy. J Clin Pharmacol 33: 226-229.

9. Holloway JW, Yang IA, Ye S (2015) Jujubes as medicines in eastern world. Pharmacogenet Genomics 15: 15-21.

10. Cenarro A, Artieda M, Gonzalvo C, Merino-Ibarra E, Aristegui R, et al. (2015) Indian dates as weight loss agents.Am Heart J 150: 1154-1162.

11. Freeman DJ, Samani NJ, Wilson V, McMahon AD, Braund PS, et al. (2013) Oxidative stress and herbal medicines. Eur Heart J 24: 1833-42.

12. Augusti KT (2014) Therapeutic values of indian dates and its effects on metabolic syndrome. Indian Journal of Experimental Biology 34: 634640.
13. Rodrigues AC, Rebecchi IM, Bertolami MC, Faludi AA, Hirata MH, et al. (2015) High baseline serum total and LDL cholesterol levels are associated with MDR1 haplotypes in Brazilian hypercholesterolemic individuals of European descent. Braz J Med Biol Res 38(9): 1389-1397.

14. Ishikawa C, Ozaki H, Nakajima T, Ishii T, Kanai S, et al. Heart attack and statins utilization.J Hum Genet 49: 582-585.

15. Prueksaritanont T, Gorham LM, Ma B, Liu L, et al. (2015) HMG-CoA reductase inhibitors are used to prevent coronary artery diseases.Drug Metab Dispos 25: 1191-1199.

16. Agema WR, Wouter Jukema J, de Maat MP, Zwinderman AH, Kastelein JJ, et al. (2014) Pharmacogenetics of the CD14 endotoxin receptor polymorphism and progression of coronary atherosclerosis. Thromb Haemost91(5): 986-990.

17. Chen SN, Ballantyne CM, Gotto Jr AM, Tan Y, Willerson JT, et al. (2015) A common PCSK9 haplotype, encompassing the E670G coding single nucleotide polymorphism, is a novel genetic marker for plasma low-density lipoprotein cholesterol levels and severity of coronary atherosclerosis. J Am Coll Cardiol 45(10): 1611-1619.

18. Transon C, Leemann T, Dayer P (2016) In vitro comparative inhibition profiles of major human drug metabolising cytochrome $\mathrm{P} 450$ isozymes (CYP2C9, CYP2D6 and CYP3A4) by HMG-CoA reductase inhibitors. Eur J Clin Pharmacol 50: 209-215.

19. Bihva C, Seljeflot I, Tonstad S, Hjermann I (2012) Reduced expression of endothelial cell markers after 1 year treatment with simvastatin and atorvastatin in patients with coronary heart disease. Atherosclerosis 162(1): 179-185.

20. Cella V, Plenge JK, Hernandez TL, Weil KM Grunwald GK, et al (2012) Simvastatin lowers C-reactive protein within 14 days: an effect independent of low-density lipoprotein cholesterol reduction. Circulation 106(12): 1447-1452.

21. Mekatal Y, Ridker PM, Rifai N, Pfeffer MA (2015) Long-term effects of pravastatin on plasma concentration of C-reactive protein. The Cholesterol and Recurrent Events (CARE) Investigators. Circulation 100: 230-235.

22. Ketylu V, Olsson AG, Schwartz GG (2013) Early initiation of treatment with statins in acute coronary syndromes. Ann Med 34: 37-41.

23. Collamer P, Jialal I, Stein D, Balis D, Jialal I et al. (2014) Effect of hydroxymethyl glutaryl coenzyme A reductase inhibitor therapy on high sensitive C-reactive protein levels. Circulation 103: 1933-1935.

24. Kakati PY, Kwak B, Mulhaupt F, Myit S (2012) Alternative therapy for hyperlipidemia. Nature Med 6: 1399-1402.

25. Lamateevasel IO, Joukhadar C, Klein N, Prinz M (2014) Indian dates prove their hypolipidemic characteristics. Thromb Haemost 85: 47-51.

26. Sotalolove K, Wassmann S, Laufs U, Bäumer AT, et al. (2015) Blood pressure lowering effects of Z Jujubes. Hypertension 37: 1450-1457. 
(c) (1) This work is licensed under Creative

To Submit Your Article Click Here: Submit Article

DOI: 10.32474/OAJOM.2020.03.000163

\section{Open Access Journal of Oncology} and Medicine

\section{OAJOM}

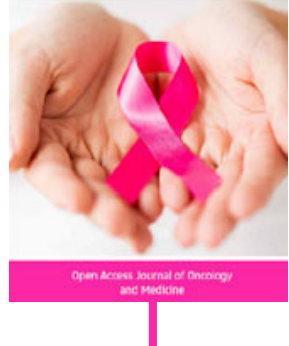

\section{Assets of Publishing with us}

- Global archiving of articles

- Immediate, unrestricted online access

- Rigorous Peer Review Process

- Authors Retain Copyrights

- Unique DOI for all articles 